B.D. $+64^{\circ} 1405$.

Farbe 6.j. Spectrum von Cl. III. a mit Banden, die im Roth und Orange gut sichtbar, im Grin etrras scliwacher sind.

B.D. $+\pi 0 \omega 0 \overline{0}=\alpha$ Draconis.

Farbe 7.j. Recht hubsches Spectrum Cl. III.a mit Banden sichtbar durch alle Farbenzonen.

B.D. +290721 .

Farbe i.jo. Nettes Colonnenspectrum ron Cl. III. a mit breiten. diffusen Banden.

B.D $+\$ \$^{0} 112=i$ Crsae ifin.

Farbe ij.j. Deutliches aber wenig brillantes Spectrum von Cl. III.a.
Schliesslich bemerke iol, dass in meiner frtheren Mittheilung in Nr. 2200 der A. N. durch ein Versehen sich ein Irrthum eingeschlichen hat, indem eins von den Spectren, namlich das von B.D $+66^{\circ} 878$. welches D'Arrest sohon fruber beobachtet hat. als neu aufgefuhrt wurde. In derselben Publication kommt aucls ein Druckfeliler vor. Der Stern $i$ Draconis ist nämlich als B.D. + 650593 bezeichnet. Statt dessen nuss: $+60^{\circ} 963$ gelesen rerden.

Lund den 21. Juni 1878.

\title{
A. CIInos.
}

Planetenbeubachtungen.

augestullt am le-fussigen Aequatoreal der Leipziger Sternwarte. mitgetheilt rou Herrn Professor C. Brulus.

16. Protogeneia.

\begin{tabular}{|c|c|c|c|c|c|c|c|c|c|c|}
\hline \multirow{2}{*}{ L8T } & \multirow{2}{*}{$\begin{array}{c}\text { Mittl. Zeit } \\
\text { Leipzig }\end{array}$} & \multicolumn{2}{|c|}{ Planet - } & \multirow{2}{*}{ A.R. app. } & \multirow{2}{*}{ Lot. f p. } & \multirow{2}{*}{ Decl. app. } & \multirow{2}{*}{ Lg.f.p } & \multirow{2}{*}{\begin{tabular}{|c|} 
Zahl der \\
Beol.
\end{tabular}} & \multirow{2}{*}{$\begin{array}{l}\text { Tgl. } \\
\text { Stcirs. }\end{array}$} & \multirow{2}{*}{$\begin{array}{c}11 . \\
\text { othactst. }\end{array}$} \\
\hline & & $\because-\infty$ & $J^{\prime}-d$ & & & & & & & \\
\hline an. 10 & $1: 216^{\mathrm{m}} 4$ & 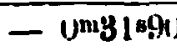 & $+4+10^{\circ} 6$ & $7^{\text {hl }}$ (ym35n-1 & 9.14 iin & $T^{\prime 2} 1^{\prime \prime} \times 3^{\prime}$ & 0.0508 & $12: 4$ & 1 & $\mathrm{p}$ \\
\hline 23 & 9.2 & $-19 . \overline{3} 1$ & +2. & (i.j) 2 s4 & y. (xitisn & +21.1549 & 51 & $1 \times: 13$ & 2 & $\mathbf{P}$ \\
\hline (1) & 111.10 .311 & -213.9 & -4.39 .5 & 6.48 .35 .46 & $8.31+n$ & +21.20 .12 & (5) 643 & $18: \cdot 1$ & $\ddot{3}$ & $\mu$ \\
\hline 28 & T.tii.20 & -11.40 .5 & $-\bar{j} . \overline{3} 3.1$ & $6: 46$ 35.27 & y $379 n$ & +-21.22 .9$. & 0.681 & $18: i i$ & 4 & $\mu$ \\
\hline
\end{tabular}

(3i) Fides.

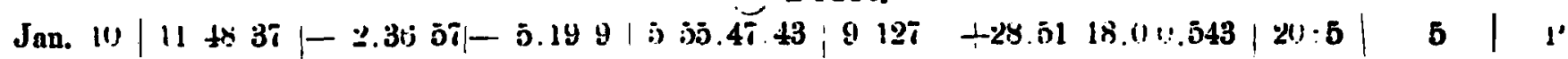

ing Ex.

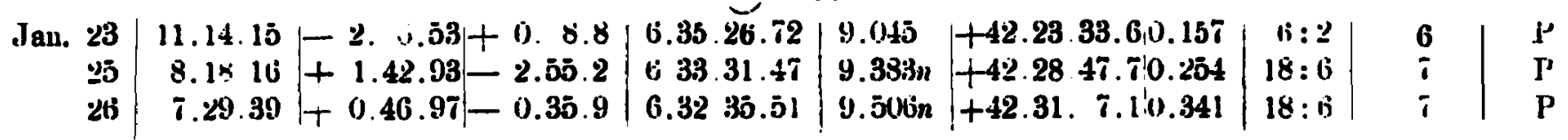

Leto.

\begin{tabular}{r|r|r|r|r|r|r|r|r|r} 
Jan. 25 & 12.4 .51 & +1.31 .25 & +2.0 .3 & 7.8 .9 .40 & 9.164 & +3.11 .57 .20 .461 & $18: 6$ & 8 & $p$ \\
26 & 9.5 .215 & $+0.44 .63 \mid+2.23 .4$ & $\tau .7 .22 .78$ & $9263 n$ & +33.12 .20 .30 .476 & $8: 3$ & 8 & $p$
\end{tabular}

(118) Amalthea.

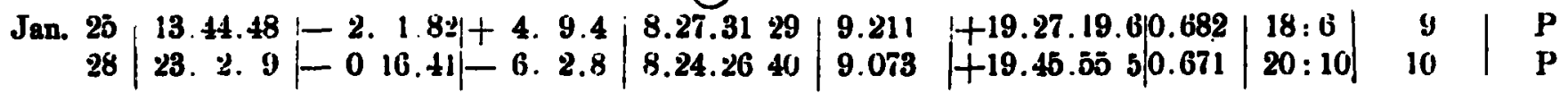

III: Cassandra.

Jan. $28|11.59 .23++0.15 .57|+0.51 .1|7.41 .49 .48| 8.918|+15.31 .5 .20 .714| 24: 12\} 11 \quad P$

(10) Rhodope.

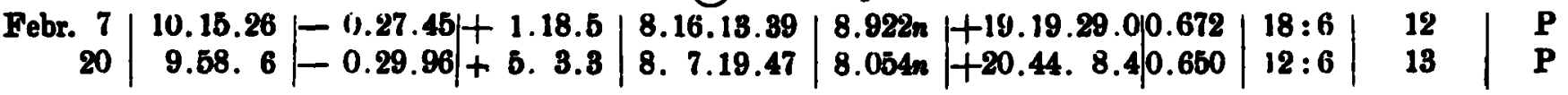




\begin{tabular}{|c|c|c|c|c|c|c|c|c|c|c|}
\hline \multirow{2}{*}{1878} & \multirow{2}{*}{$\begin{array}{c}\text { Mittl. Zeit } \\
\text { Leipzig }\end{array}$} & \multicolumn{2}{|c|}{ Planet - } & \multirow{2}{*}{ A.R. app. } & \multirow{2}{*}{ Lg. f. p. } & \multirow{2}{*}{ Decl. app. } & \multirow{2}{*}{ Lg.f.p. } & \multirow{2}{*}{\begin{tabular}{|c|}
$\begin{array}{c}\text { Zahl der } \\
\text { Beob. }\end{array}$ \\
\end{tabular}} & \multirow{2}{*}{$\begin{array}{l}\text { Vergl.- } \\
\text { Stern. }\end{array}$} & \multirow{2}{*}{$\begin{array}{c}\text { Be- } \\
\text { obacht. }\end{array}$} \\
\hline & & $\overline{a-a}$ & $\delta$ & & & & & & & \\
\hline
\end{tabular}

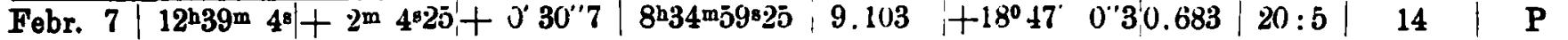

Febr.

\begin{tabular}{|c|c|}
\hline ebr. & \\
\hline 1. & $3.30 .49+1.8 .0$ \\
\hline 18 & $1.1195-6.34 .4$ \\
\hline 19 & $9.44 .34+0.27 .85+5.31 .5$ \\
\hline 20 & $1117.33-1.51 .63+3.30 .6$ \\
\hline Murz 3 & $10.49 .8+0.2 \overline{5} .27+1 . \overline{\mathbf{5}} 7.1$ \\
\hline 19 & $11.16 .51+1.46 .54+2.27 .3$ \\
\hline
\end{tabular}
9.59.3.47

8.836 9.51 .15 .35

9.175 $+12.8 \cdot 14.5 \cdot 0 \cdot 746$ 9.50 .31 .26 $9.49 .46 .46^{\circ}$ 9.42 .34 .28 9.35 .47 .27

$9421 n+14.18 .10 .70 .754$ $9.304 n+14.30 .16 .60 .739$ $8.688 n+14.42 .47 .30 .721$ $8.088 n+16.42 .38 .20 .698$ $9.176+18.59 .8 .210 .685$

$28: 7$
$16: 4$
$20: 5$
$18: 6$
$18: 6$
$17: 6$
$9: 3$

\begin{tabular}{l|l}
15 & $\mathbf{P}$ \\
16 & $\mathbf{P}$ \\
17 & $\mathbf{P}$ \\
17 & $\mathbf{P}$ \\
18 & $\mathbf{P}$ \\
19 & $\mathbf{P}$ \\
20 & $\mathbf{P}$
\end{tabular}

(120) Antigone.

Febr. 12

14. 0.24 $0.5 .45+4.11 .4 \mid 10.1 ! .50 .37$

iii, Siruna.

Febr, 18

\section{$9.35 .33|-2.44 .42-3.245| 9.56 .45 .4$ \\ 9305}

$|-1854.57 .60 .704|$

$20: 5$
$20: 5$

\begin{tabular}{l|l}
24 & $\mathbf{P}$ \\
24 & $\mathbf{P}$
\end{tabular}

(7) Niobe.

Febr. 18

März 9
$11 . \overline{2} 2$.
$5.59 .60+0.11 .6$
(1) 19.43
4.10

12

8. $4 \cdot 25-0.47 .64+4.7 .1957 .23 \times 8$

$8.719 n|-3.17 .26 .0| 0.856$

\begin{tabular}{r|l}
$12: 3$ & 25 \\
$18: 6$ & 26 \\
$9: 3$ & 26
\end{tabular}

25
26
26

$$
\begin{aligned}
& \mathbf{P} \\
& \mathbf{P} \\
& \mathbf{P} \\
& \mathbf{P} \\
& \mathbf{P} \\
& \mathbf{P}
\end{aligned}
$$

17:1,

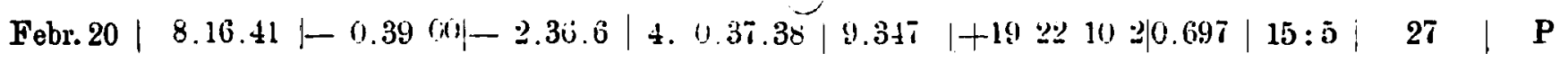

\begin{tabular}{rl|l|l|l|l|l|l|l|l|l|l|l|l} 
März & 3 & 13.19 .20 & -1.23 .68 & -3.57 .4 & 9.57 .17 .25 & 4.309 & -15.5 .12 .80 .734 & $18: 6$ & 28 & $P$ \\
& 4 & 11.14 .21 & +0.37 .61 & -3.22 .1 & 9.55 .31 .49 & 5.126 & +15.9 .15 .7 & 0.715 & $15: 5$ & 29 & $\mathbf{P}$ \\
& 5 & 1237.1 & -0.14 .58 & +1.24 .1 & 9.55 .34 .29 & 9.191 & +15.14 .1 .9 & 0.725 & $20: 5$ & 29 & $\mathbf{P}$
\end{tabular}

Mittlere Oerter der Vergleichsterne für 1878.0.

\begin{tabular}{|c|c|c|c|}
\hline $\mathbf{N r}$. & (Wolfers) & $\delta$ (Auwers) & Autorität. \\
\hline 1 & $7^{\text {h }} 1^{\mathrm{m}} 4^{\mathrm{s}}$ & $+21^{\circ} 3^{\prime} 20^{\prime \prime} 0$ & Polaer Mer. Beob. \\
\hline 2 & 6.500 .51 .44 & $+21.16 \cdot 9.0$ & Weisse II $6^{\mathrm{h}} 1493$. \\
\hline 3 & 6.50 .46 .94 & +21.24 .48 .2 & 6.1489. \\
\hline 4 & 6. $4 \overline{0} . \overline{5} .31$ & $|+21.27 .57 .9|$ & 6. 135̄o. \\
\hline $\mathbf{5}$ & 5.58 .21 .62 & +28.56 .30 .1 & ј. 1869. \\
\hline 6 & 6.37 .30 .28 & +42.23 .16 .9 & ن. 1087. \\
\hline 7 & 6.31 .45 .59 & $+42.31 .34 .3 \mid$ & b 885. \\
\hline 8 & 7. 6.35 .39 & $+33.9 .52 . \overline{0}$ & Bon." Beob. VI + 3301490. \\
\hline 9 & 8.29 .39 .58 & +19.23 .13 .0 & Rümker I 2578. \\
\hline 10 & 8.24 .40 .25 & +19.52 .0 .9 & . 2548. \\
\hline 11 & 7.41 .31 .46 & $|+15.30 .14 .1|$ & Bon. Beob. VI $+15^{\circ} 1665$. \\
\hline
\end{tabular}




\begin{tabular}{|c|c|c|c|}
\hline Nr. & $\alpha$ (Wolfers) & $\delta$ (Auwers) & Autoritat. \\
\hline 12 & $8^{\mathrm{h}} 16^{\mathrm{m}} 38^{\mathrm{s} 24}$ & $+19^{\circ} 18^{\prime} 12^{\prime \prime} 6$ & Anschluss an Lalande 16516. \\
\hline 13 & 8. 7.46 .83 & +20.39 .6 .1 & Weisse II $\gamma^{b} l 16$. \\
\hline 14 & $\begin{array}{r}8.32 .52 .59 \\
52.18\end{array}$ & $\mid \begin{array}{r}+18.46 .32 .3 \\
33.6\end{array}$ & $\begin{array}{l}\text { Weisse II } 8 \text { th8s. } \\
\text { Astr. Nachr. B. } 43 \text { S. } 113 .\end{array}$ \\
\hline & 8.32 .82 .39 & $\mid+18.46 .32 .9$ & $\frac{W+A . N .}{2}$ \\
\hline 15 & 10. 1.42 .89 & +12.5 .55 .0 & Bon. Beob. + 12"2146. \\
\hline 16 & $\begin{array}{r}9.58 .55 .53 \\
55.97 \\
5 \overline{5} .82 \\
\overline{50} .5 .2\end{array}$ & $\left|\begin{array}{r}713.53 .1 \\
51.4 \\
52.1 \\
52.2\end{array}\right|$ & $\begin{array}{l}\text { Bon. Mer. Beob. (Astr. Nachr. B. } 58 \text { S. 9.) } \\
\text { Berl. Mer. Beob. (Astr. Nachr. B. } 58 \text { S. 231·) } \\
\text { Washington Cat. 4199. } \\
\text { Bon. Beob. VI. + } 13^{\circ} 2202 .\end{array}$ \\
\hline & $9.58 .0 \overline{0} .71$ & +13.7 .52 .2 & $\frac{\text { A.N. }+ \text { A.N. }+ \text { IV.C. }+ \text { B.B. }}{4}$ \\
\hline 17 & $9.50 \cdot 0.78$ & +14.24 .54 .0 & Berl. Mer. Beob. (Astr. Nachr. B. 58 S. 231.) \\
\hline 18 & 9.51 .35 .45 & +14.39 .25 .8 & Bon. Beob. VI. + 1402172. \\
\hline 19 & 9.42 .6 .30 & +16.40 .49 .2 & Weisse II $9^{\mathrm{h}} 862$. \\
\hline 20 & 9.33 .58 .06 & +16.56 .47 .5 & Anschluss an Weisse II $9 \mathrm{~h} \mathrm{~h} 76$. \\
\hline 21 & 10.11 .53 .27 & +14.32 .354 & Weisse $I 10^{\mathrm{h}} 170$ \\
\hline 22 & 10.11 .35 .91 & +15.24 .43 .6 & Rumker I $10^{\text {h }}$ Nachtrag. \\
\hline 23 & 10. 6.42 .40 & $\mid+15.46 .19 .9$ & 3084 \\
\hline 24 & 9.59 .27 .12 & +19.3 .31 .2 & Weisse II $9^{\mathrm{h}} 1245$. \\
\hline 25 & 10.23 .39 .73 & -3.17 .27 .0 & Weisse $110 \mathrm{l} 398$ \\
\hline 26 & $9.58 \cdot 9.11$ & -6.22 .5 .4 & $9 \mathbf{h}_{1} 230$ \\
\hline $2 \tau$ & 4. $\begin{array}{r}1.15 .30 \\
15.04 \\
\end{array}$ & $\begin{array}{r}+1924.36 .8 \\
37.2 \\
\end{array}$ & $\begin{array}{l}\text { Weisse II } 3^{\mathrm{h}} 128 \mathrm{~g} . \\
\text { Rumker I } 1091 .\end{array}$ \\
\hline & 4.1 .15 .17 & +1924.37 .4 & $\frac{\mathrm{W}+\mathrm{R}}{2}$ \\
\hline 28 & $9.5 \overline{5} . \overline{\mathrm{y}} 0.88$ & +15.9 .19 .5 & Lalande 19593. \\
\hline 29 & $9 . \overline{5} . \overline{1} 1.19$ & $|+15.12 .47 .2|$ & Bon. Beob. VI. + 1502155 \\
\hline
\end{tabular}

\section{Vergleichung der Beobachtungen}

mit den Ephemeriden des Berlinerastron. Jahrbuches (Beob.-Rechn.)

(3i. Fides.

Jan. $10 \quad \Delta^{\alpha}=-10^{\mathrm{s}} 78 \quad \Delta \delta=-6^{\prime \prime} 8$

$$
\text { (68) Leto. }
$$$$
\text { Jan. } \begin{array}{rlr}
25 & +35.59 & -47.2 \\
26 & +35.56 & -45.5
\end{array}
$$

(113) Amalthea.

$$
\begin{array}{rlr}
\text { Jan. } 25 & +22.37 & -54.7 \\
28 & +22.45 & -56.4
\end{array}
$$

III. Cassandra.

$$
\text { Jan. } 28 \quad-34.23 \quad+67.5
$$

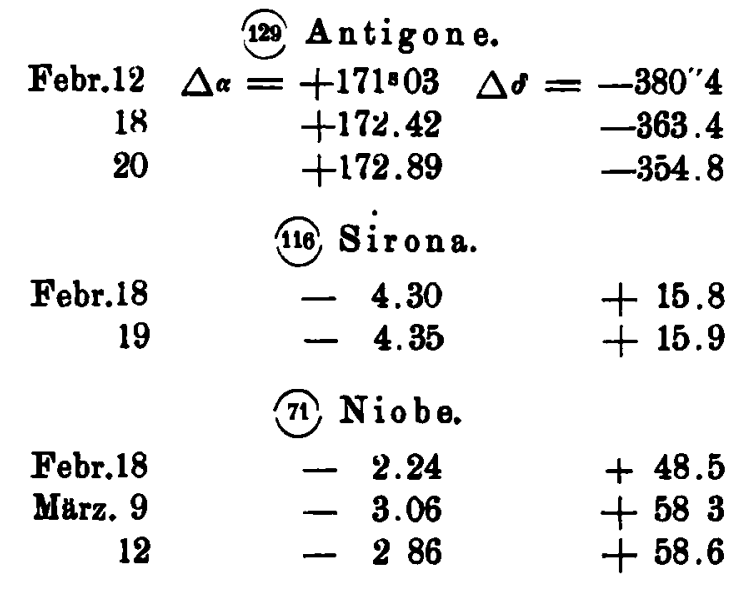

